\title{
Identification of long non-coding RNA SCARNA9L as a novel molecular target for colorectal cancer
}

\author{
JIE CHAI $^{1,2}$, JIANBO ZHANG $^{3}$, DALI HAN $^{4}$, WEI DONG $^{4}$, LI HAN $^{5}$, \\ LEI ZOU ${ }^{2}$, BIN FENG $^{6}$, BAOSHENG $\mathrm{LI}^{4}$ and WANLI MA ${ }^{7}$
}

\author{
${ }^{1}$ Department of Internal Medicine-Oncology, Tianjin Medical University, Tianjin 300070; Departments of ${ }^{2}$ Gastrointestinal \\ Surgery, ${ }^{3}$ Pathology, ${ }^{4}$ Radiation Oncology and ${ }^{5}$ Internal Medicine-Oncology, Shandong University Affiliated Shandong Cancer \\ Hospital and Institute, Jinan, Shandong 250117; ${ }^{6}$ Department of Internal Medicine-Oncology, Affiliated Hospital of \\ Shandong Academy of Medical Sciences; ${ }^{7}$ Department of Orthopedics, The Second Hospital of \\ Shandong University, Jinan, Shandong 250033, P.R. China
}

Received July 17, 2019; Accepted March 26, 2020

DOI: 10.3892/ol.2020.11661

\begin{abstract}
The aim of the present study was to analyze the microarray data of human colorectal cancer (CRC) tissues and identify novel therapeutic targets for CRC. Microarray analysis from the GSE73360 and GSE84984 datasets was performed to identify novel long non-coding RNAs (lncRNAs) that were differentially expressed in human CRC tissues. Additionally, small interfering RNAs were used to deplete the expression of the indicated lncRNAs in cells. Colony-formation, wound-closure, and transwell assays were performed on CRC cells to assess their proliferation and migration capacities. Through microarray analysis, SCARNA9L, SLMO2-ATP5E and LOC100132062 were identified as differentially expressed lncRNAs in CRC tissues. The present study demonstrated that the ablation of SCARNA9L inhibited cell proliferation and arrested the cell cycle of SW480 and SW620 CRC cells. Additionally, depletion of SCARNA9L restrained the migration of CRC cells in vitro. Overall, the present study investigated the potential involvement of SCARNA9L in CRC and suggests SCARNA9L as a potential biomarker.
\end{abstract}

Correspondence to: Dr Baosheng Li, Department of Radiation Oncology, Shandong University Affiliated Shandong Cancer Hospital and Institute, 440 Jiyan Road, Jinan, Shandong 250117, P.R. China

E-mail: baoshli1963@163.com

Dr Wanli Ma, Department of Orthopedics, The Second Hospital of Shandong University, 247 Beiyuan Street, Jinan, Shandong 250033, P.R. China

E-mail: mawanli2000@126.com

Abbreviations: CRC, colorectal cancer; lncRNA, long non-coding RNAs; GEO, Gene Expression Omnibus; DEGs, differentially expressed genes; $\mathrm{NC}$, negative control

Key words: CRC, SCARNA9L, microarray, proliferation, migration

\section{Introduction}

Colorectal cancer (CRC), which is also known as bowel and colon cancer, originates from the colon or rectum (1-3). According to the analysis of the prevalence of malignant tumors in China in 2015, released by the National Cancer Center in 2019, CRC ranks the third most common cancer among the top 10 malignant tumors $(4,5)$. The number of patients with CRC and their mortality has reached a high level worldwide in the past years (3). Although advances in surgery, radiation therapy and chemotherapy have been recently made for the treatment of CRC, due to the fast-growing nature and high rate of metastasis of this cancer, the 5-year survival rate of CRC is still low, at only 14\% (6-8). Recently, targeted therapy has been revealed as a promising prospect for CRC treatment (9). Although several therapeutic targets of CRC have been developed to combat this malignant tumor, more effective therapeutic targets are urgently required (10).

Long non-coding RNAs (lncRNA) are defined as transcripts that are not translated into proteins (11). LncRNAs have multiple cellular and physiological functions, such as combating oxidative stress $(12,13)$. A large number of studies have indicated that lncRNAs are associated with diverse human diseases, such as myelodysplastic syndromes, Alzheimer's disease and fatty liver disease (14-16). In addition, the IncRNA Neat1 may contribute to the activation of inflammasomes in macrophages (17).

Notably, the potential effects of lncRNAs on multiple types of cancer have been revealed in recent decades (18). Additionally, studies have recently shown that IncRNAs may be abnormally expressed in tumor tissues (19). Furthermore, IncRNAs are involved in the progression of bladder cancer, breast cancer and glioma $(18,20)$. LncRNAs were also demonstrated to affect the proliferation, apoptosis, migration and invasion of tumor cells through different mechanisms (18). The lncRNA ABHD11-AS1 facilitates thyroid carcinoma progression via the PI3K/AKT pathway (21). Additionally, the IncRNA GAS6-AS1 promotes gastric cancer progression by targeting GAS6 (22). However, novel lncRNAs that can regulate the development of CRC still need to be identified. 
Using a microarray analysis, the present study aimed to identify upregulated genes in human CRC tissues, which may be involved in the progression of CRC. Additionally, the present study aimed to reveal the signaling pathways and the core lncRNAs with potential to affect CRC progression. Through a series of in vitro assays, a novel and potential oncogene involved in CRC progression was identified.

\section{Materials and methods}

Samples. A total of 36 CRCs and the corresponding adjacent ( $2 \mathrm{~cm}$ away from the tumor border) surgical specimens in the present study were collected between November 2018 and May 2019, with complete clinicopathological data collected at The Second Hospital of Shandong University (Jinan, China). In the present study, patients had only received surgical treatment, and patients who had received chemotherapy and/or targeted therapy were excluded. The patients ranged in age between 35 and 65 years (mean age, 52 years), with 20 males and 16 females. All studies were approved by the Ethics Committee of The Second Hospital of Shandong University, and informed written consent was obtained from all patients.

Sequences of small interfering RNAs (siRNAs) and quantitative $P C R$ primers. The siRNA sequence targeting SCARNA9L was: 5'-CGGUCUACC UGAUGCAUGAUCUCUA-3'. The quantitative PCR primer sequences were as follows: SCARNA9L forward, 5'-ATAAAGGTAGCAGTTGTAGGA ATG-3'; SCARNA9L reverse, 5'-CTTCATAGTTACAAA GGTCAGTCG-3'; GAPDH forward, 5'-CGACCACTTTGT CAAGCTCA-3'; and GAPDH reverse, 5'-GGTTGAGCACAG GGTACTTTATT-3'.

Bioinformatics analysis. CRC gene expression microarrays were searched using the search term 'Colorectal cancer' in the gene expression omnibus (GEO) database (https://www.ncbi.nlm. nih.gov/geo/). GSE73360 and GSE84984 datasets were used to screen the differentially expressed lncRNAs in CRC (23-27). The process included: Comparison analysis by limma R package (28) (version:3.36.5; http://bioinf.wehi.edu.au/limma); Gene Ontology (GO; http://geneontology.org/); analysis through statistical method, by two side Fisher's exact test (29); pathway analysis through two side Fisher's exact test (30) (https://www.genome. $\mathrm{jp} / \mathrm{kegg} /$ ); coexpression network analysis using $\mathrm{R}$ function (Cytoscape; version, 3.6.0; https://cytoscape.org/) (31); and global signal transduction network (32) (version, 3.6.0; https://cytoscape. org/; https://www.genome.jp/kegg/).

Scatter diagrams were plotted. Additionally, the limma package v3.36.5 (http://master.bioconductor. org/packages/release/bioc/html/limma.html) was used to screen differentially expressed genes (DEGs), with $\mathrm{P}<0.05$ and $\log$ fold-change (FC) $>2$ set as the threshold. The heatmap of differentially expressed lncRNAs was drawn using the Heatmap Package v3 (https://CRAN.R-project.org/package=heatmap3). Pathway enrichment analysis and coexpression analysis were performed, according to the GSE73360 and GSE84984 datasets, with a false discovery rate $(\mathrm{FDR})<0.05$ considered significant. Data on the lncRNA expression were obtained from the Oncomine database (www.oncomine.org). The expression data are available from the GEO database (www.ncbi.nlm.nih.gov/geo) (23-27).
Cell culture and transfection. SW480 and SW620 human CRC CRC cells were obtained from The Cell Bank of Type Culture Collection of the Chinese Academy of Sciences. SW480 and SW620 cells were maintained in DMEM culture medium (Gibco; Thermo Fisher Scientific, Inc.), and supplemented with $10 \%$ fetal bovine serum (FBS; Gibco; Thermo Fisher Scientific, Inc.) in a $5 \% \mathrm{CO}_{2}$ incubator at $37^{\circ} \mathrm{C}$.

Both SW480 and SW620 CRC CRC cells were transfected with control (scrambled siRNA) or the aforementioned siRNA targeting SCARNA9L (50 nM; Guangzhou RiboBio Co., Ltd.) using Lipofectamine ${ }^{\mathrm{TM}} 3000$ (Thermo Fisher Scientific, Inc.), according to the manufacturer's instructions. The in vitro assays were performed $48 \mathrm{~h}$ after transfection.

Reverse transcription-quantitative (RT-q)PCR assay. TRIzol reagent (cat. no. 9108; Takara Bio, Inc.) was used to extract total RNA from both SW480 and SW620 cells. Total RNA was subsequently reverse transcribed at $37^{\circ} \mathrm{C}$ for $1 \mathrm{~h}$ using M-MLV reverse transcriptase (cat. no. M1701; Promega Corporation) and using a cDNA synthesis system (cat. no. RR037A; Takara Bio, Inc.), including oligo DT, Random 6-mers (100 $\mu \mathrm{M})$, primers, 5X PrimeScript buffer, PrimeScript RT Enzyme Mix I and DEPC water. qPCR was performed using SYBR Premix Ex Taq ${ }^{\mathrm{TM}}$ (cat. no. RR820A; Takara Bio, Inc.) with the following thermocycling conditions: $94^{\circ} \mathrm{C}$ for $3 \mathrm{~min}$, followed by 35 cycles of $94^{\circ} \mathrm{C}$ for $40 \mathrm{sec}, 55^{\circ} \mathrm{C}$ for $1 \mathrm{~min}$ and $72^{\circ} \mathrm{C}$ for $1 \mathrm{~min}$, and finally $72^{\circ} \mathrm{C}$ for $10 \mathrm{~min}$. The relative expression level of the indicated RNAs was normalized to the expression of GAPDH using the $2^{-\Delta \Delta \mathrm{Cq}}$ method (33).

Colonyformation assays. Approximately 1,000 NC-transfected cells or human CRC cells treated with the indicated siRNA treatment were seeded into 6-well culture plates and incubated with $5 \% \mathrm{CO}_{2}$ at $37^{\circ} \mathrm{C}$ for 7 days. The medium was replaced with fresh complete DMEM (with $10 \%$ FBS) every two days. After 7 days, the cells were washed with PBS twice, fixed with paraformaldehyde for $30 \mathrm{~min}$ at room temperature, and stained with $1 \%$ crystal violet at room temperature for $15 \mathrm{~min}$, washed with PBS and observed using the Olympus CX31 light microscope (magnification, x10). Subsequently, the number of colonies was manually counted.

Cell cycle assays. The cell cycle distribution was detected by using a cell cycle kit (Beijing 4A Biotech Co., Ltd.). In brief, NC-transfected or indicated siRNA-treated CRC cells were fixed with $95 \%$ ethanol at $-20^{\circ} \mathrm{C}$ for $24 \mathrm{~h}$ and incubated with $0.4 \mathrm{ml}$ of propidium iodide $(200 \mu \mathrm{g} / \mathrm{ml})$ for $30 \mathrm{~min}$ at room temperature prior to analysis by flow cytometry.

Wound-closure assays. Both SW480 and SW620 CRC CRC cells were transfected with siRNAs and grown to confluent monolayers. Subsequently, a scratch was generated using a $10-\mu 1$ pipette tip. Cell debris was washed twice using PBS, and serum-free medium was added to induce wound healing. Images of the wounds were captured at 0 and $24 \mathrm{~h}$ using the Olympus CX31 light microscope (magnification, $\mathrm{x} 10$ ), and the percentage of wound closure was measured. The width of the wound was indicated using lines at the edge of the wound, and the closure percentage was measured as the healing width divided by the original wound width. 

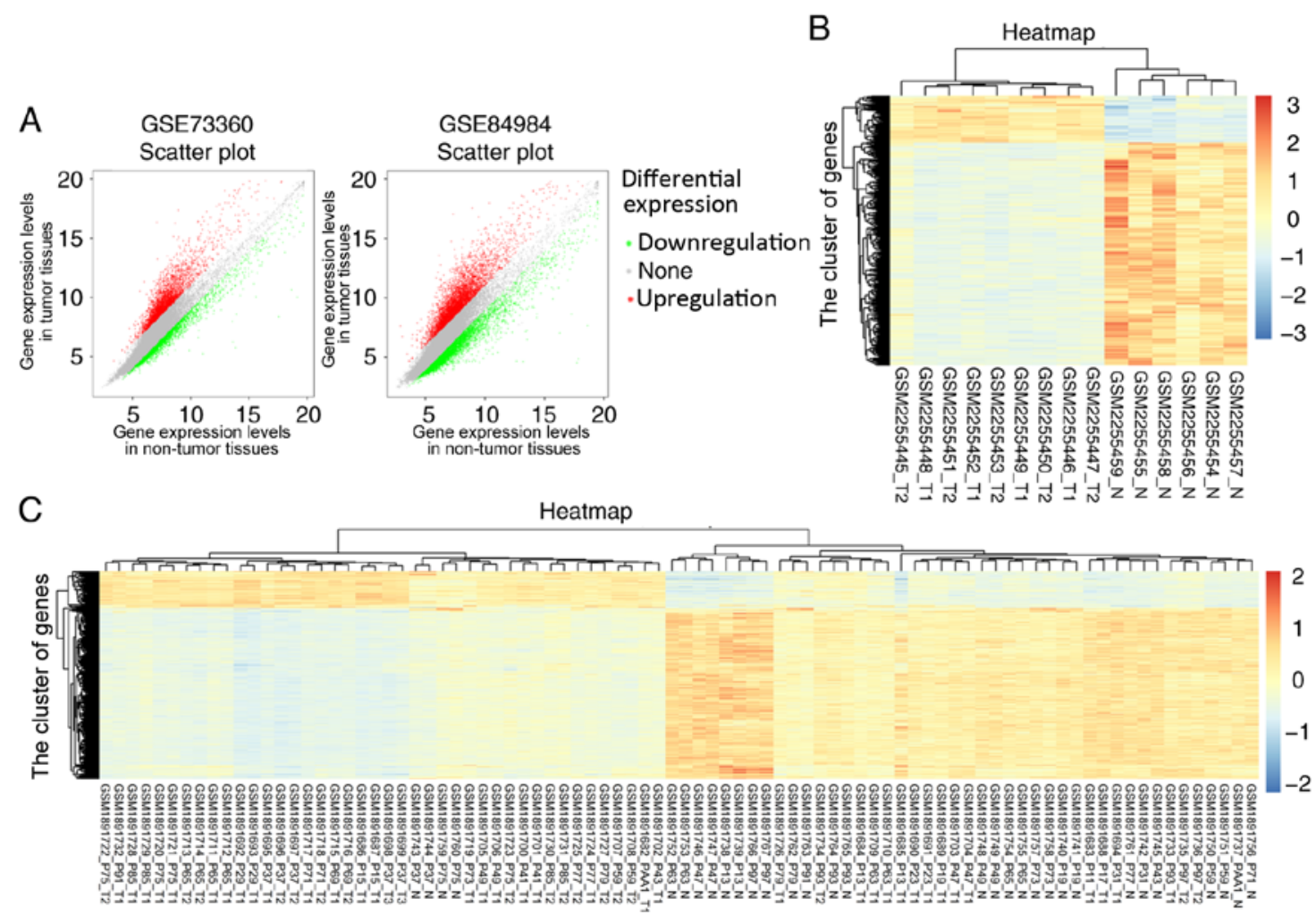

Figure 1. Identification of novel differentially expressed lncRNAs in colorectal cancer via microarray analysis. (A) Scatter diagrams of all lncRNAs from the GSE73360 and GSE84984 datasets. Heat map showing the differential expression of 1ncRNAs from the (B) GSE73360 and (C) GSE84984 datasets. The degree of gene expression differentiation is shown as red (upregulation) or blue (downregulation). lncRNAs, long non-coding RNAs.

Transwell assays. SW480 and SW620 cells were transfected with siRNAs for $48 \mathrm{~h}$ and then trypsinized and resuspended in serum-free DMEM (Gibco; Thermo Fisher Scientific, Inc.). A total of $1 \times 10^{5}$ cells in $100 \mu$ l DMEM without FBS were then added to the upper chambers of the Transwell inserts (Millicell; Merck KGaA) and allowed to migrate toward the bottom of the chambers, which contained DMEM with $10 \%$ FBS. After $24 \mathrm{~h}$, the remaining cells in the upper chamber were removed, and cells on the underside were fixed in $4 \%$ paraformaldehyde for $30 \mathrm{~min}$ at room temperature, stained with $0.1 \%$ crystal violet for $30 \mathrm{~min}$ at room temperature and captured using an Olympus type light microscope sz30. Quantification of the migrated cells was performed by counting cell numbers.

Statistical analysis. GraphPad Prism 6.0 software (GraphPad Software, Inc.) was used for statistical analysis. All data in this study are presented as the mean \pm standard deviation (SD). Statistical significance was calculated using a one-way analysis of variance following by the Fisher's Least Significant Difference post hoc test. Paired t-test was used to compare the expression of lncRNAs in tumor tissues and normal tissues. Additionally, the expression of SLMO2-ATP5E and LOC100132062, and the effects of siRNAs on CRC cell proliferation and migration were analyzed using unpaired t-tests. $\mathrm{P}<0.05$ was used to indicate a statistically significant difference.

\section{Results}

Identification of novel differentially expressed lncRNAs in $C R C$ via microarray analysis. Microarray analysis was used to screen CRC-associated DEGs and predict the lncRNAs involved in CRC progression. Analysis of the CRC gene expression datasets GSE73360 and GSE84984 was performed. The microarray data of all lncRNAs in CRC tissues were extracted from the GEO dataset. $\mathrm{R}$ language was used to screen for differentially expressed lncRNAs from the CRC gene expression datasets, and the scatter diagrams of all lncRNAs from the GSE73360 and GSE84984 datasets are shown in Fig. 1A. In total, 2233 differentially expressed lncRNAs were screened from GSE73360, and 6749 DEGs were screened from GSE84984, based on $\mathrm{P}<0.05$ and $\mathrm{FC}>2$.

Furthermore, the heatmaps of differentially expressed lncRNAs that were screened from the GSE73360 and GSE84984 datasets are shown in Fig. 1B and C, respectively. According to the results of the heatmaps, SCARNA9L, SLMO2-ATP5E, and LOC100132062 were highly expressed in CRC, in both the GSE73360 and GSE84984 datasets. To the best of our knowledge, there are no studies on the effects of these IncRNAs on CRC; therefore, the differential expression of SCARNA9L, SLMO2-ATP5E, and LOC100132062 and their possible regulatory mechanisms in CRC progression were investigated in the present study.

Interestingly, according to the KEGG pathway analysis of GSE73360 and GSE84984, several pathways were notably affected (including those upregulated and downregulated) in CRC tissues (Fig. 2A and B). Several genes in the indicated pathways, such as tyrosine metabolism and fatty acid degradation pathways, were downregulated, suggesting effects on cell metabolism and CRC progression (Fig. 2A). Additionally, in the analysis of enrichment pathways for upregulated genes in the GSE73360 
A

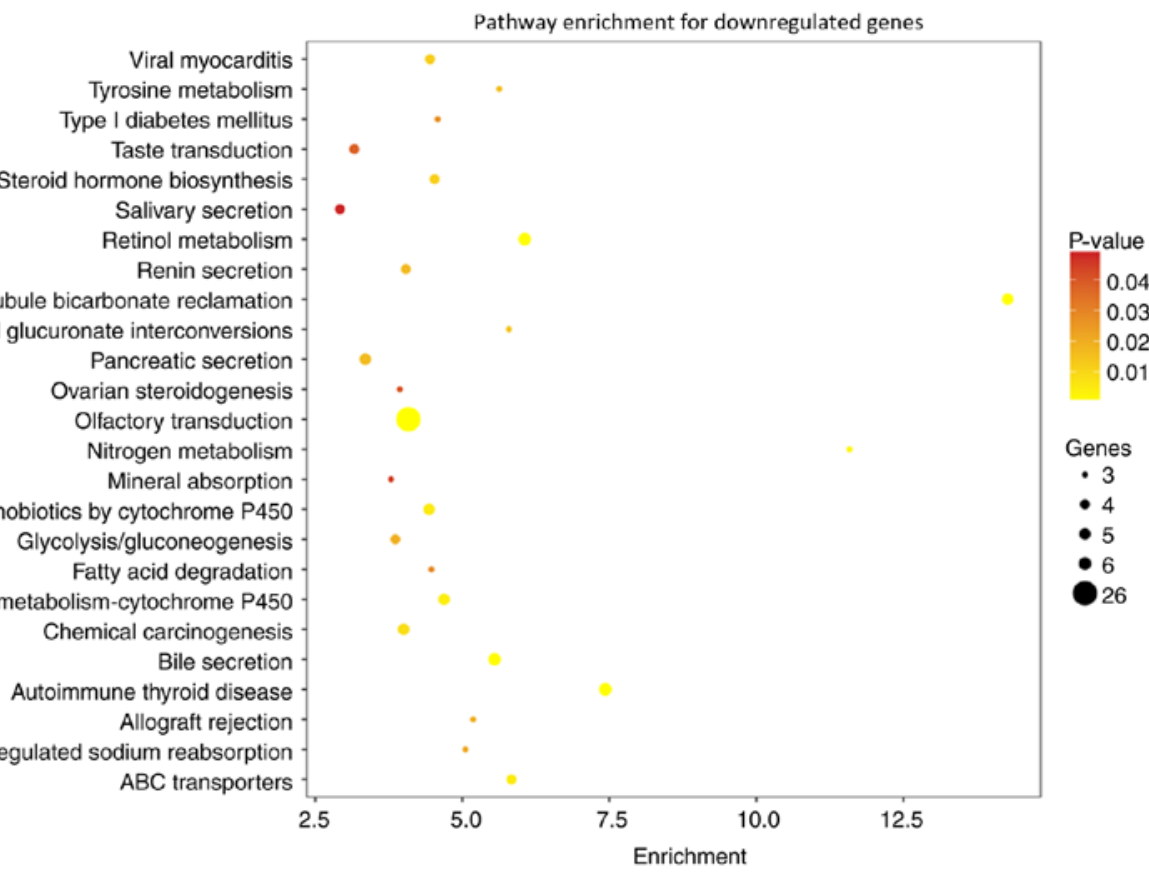

B

Pathway enrichment for upregulated genes

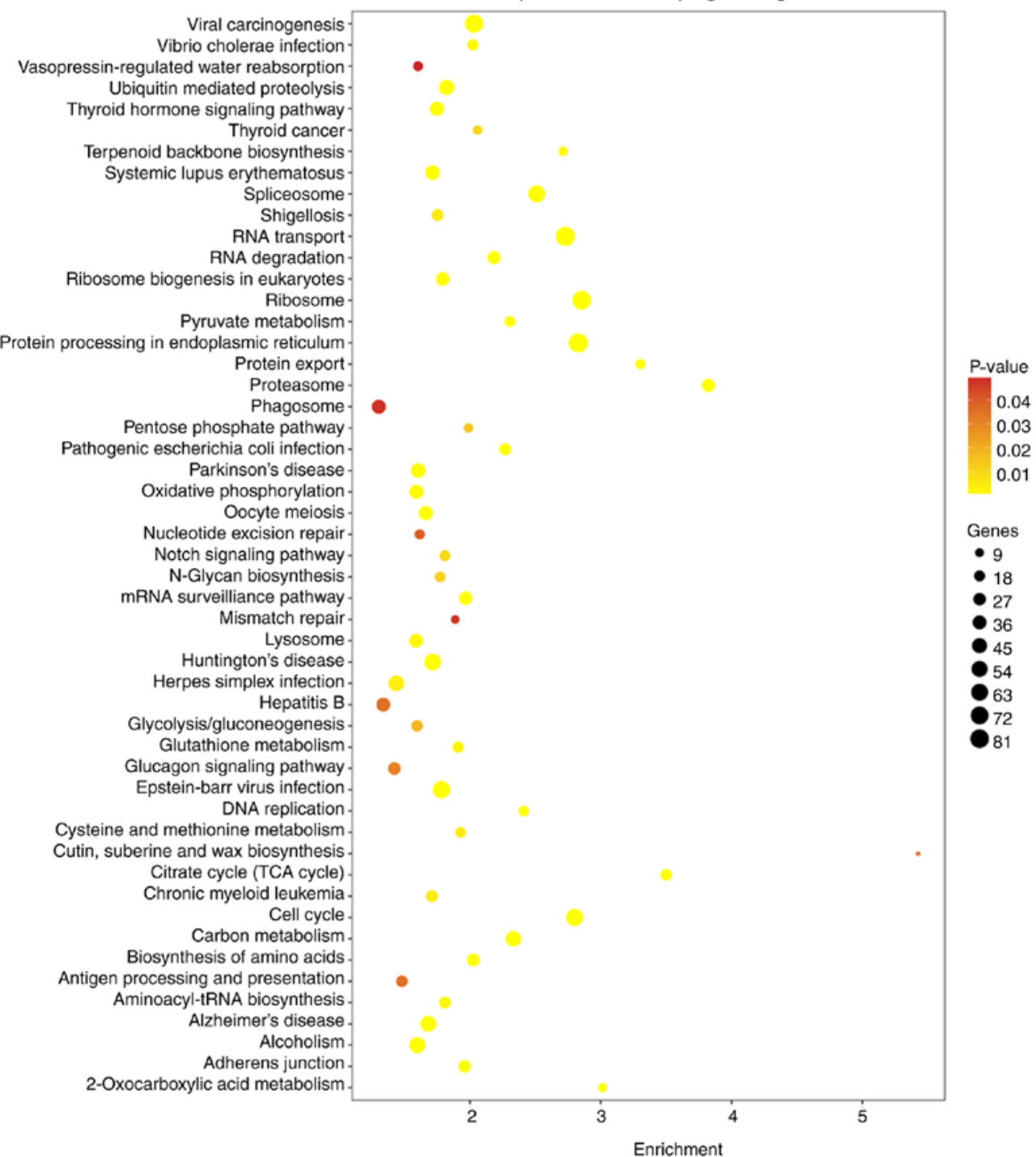

Figure 2. Analysis of enrichment pathways according to the GSE73360 and GSE84984 datasets. The enrichment pathways for (A) downregulated genes and (B) upregulated genes. The P-value indicated by different shades of color represents the significance. The different sizes of the circles $(3,4,5,6$ and 26$)$ represent the identified gene numbers in this group. 


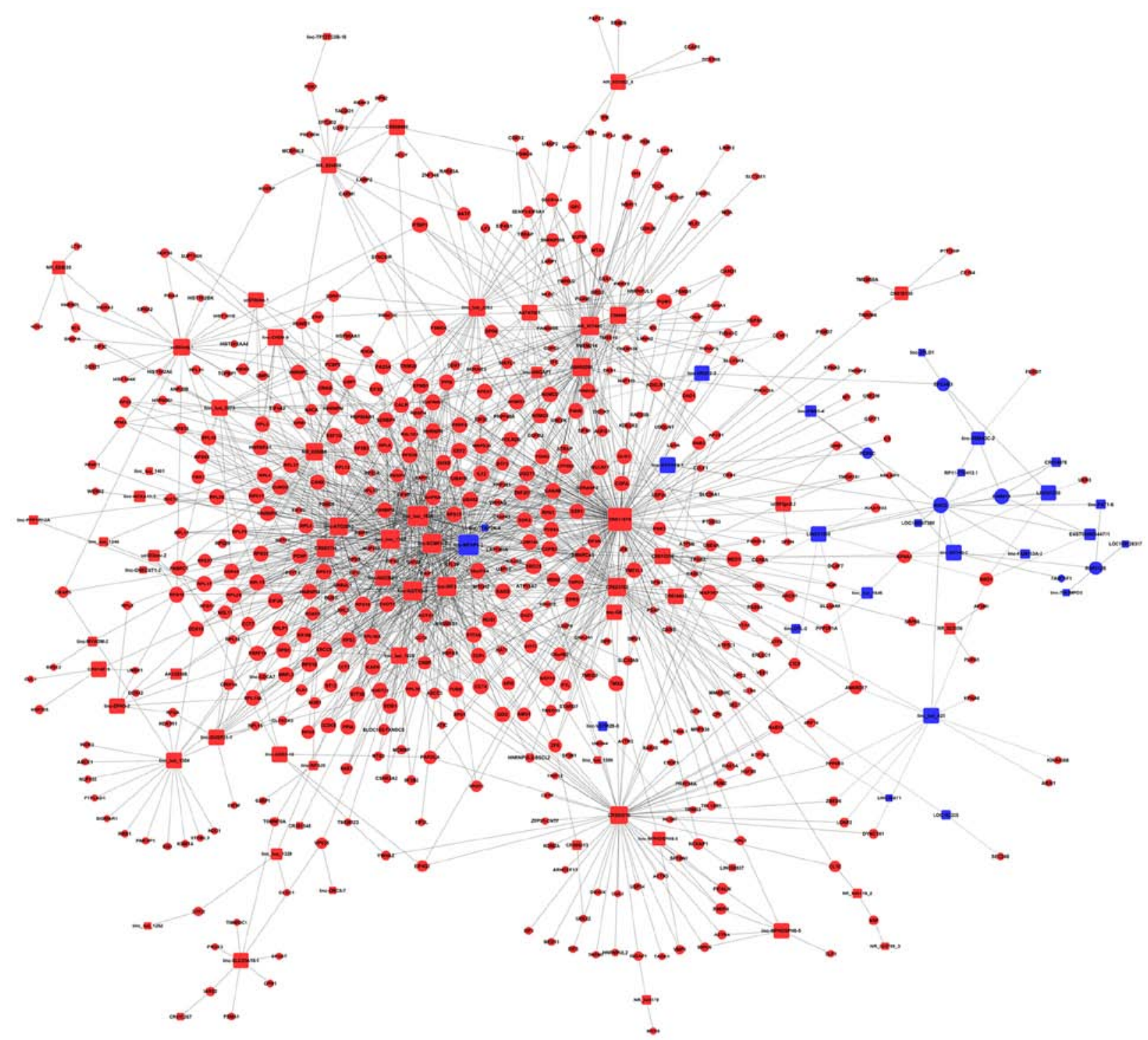

Figure 3. Coexpression network of lncRNAs according to the GSE73360 and GSE84984 datasets. Red represents the non-differentially expressed lncRNAs, and blue represents the differentially expressed lncRNAs. IncRNAs, long non-coding RNAs.

and GSE84984 datasets, the cell cycle, adherent junctions, and mismatch repair pathways, which have the potential to direct affect CRC progression, were notably upregulated (Fig. 2B).

Subsequently, further coexpression network analysis on GSE73360 and GSE84984 datasets were performed to identify the key lncRNAs involved in the regulation of CRC progression (Fig. 3). Interestingly, the data revealed that multiple core lncRNAs affected the expression of key proteins involved in the regulation of cancer progression, such as lnc-MFAP4-2, lnc-TFAP-2A-4, and lnc-KRTAP9-1 (Fig. 3), and the precise mechanisms need further study. Furthermore, three obviously upregulated lncRNAs, SCARNA9L, SLMO2-ATP5E, and LOC100132062, were identified from the coexpression network analysis (Fig. 3).

Taken together, three lncRNAs, SCARNA9L, SLMO2ATP5E, and LOC100132062, were notably upregulated and have the potential to be involved in CRC progression.

Knockdown of SCARNA9L inhibits the proliferation of SW480 and SW620 cells. The potential involvement of the three identified IncRNAs, SCARNA9L, SLMO2-ATP5E and LOC100132062, in the progression of CRC was further explored.

siRNAs targeting the indicated lncRNAs were designed and transfected into two human CRC cell lines, SW480 and SW620 cells, to inhibit its expression. Cell proliferation is critical in cancer progression. Therefore, colony-formation assays were performed to investigate whether these three lncRNAs affected the proliferation of CRC cells. As was confirmed by RT-qPCR assays, the expression levels of SLMO2-ATP5E and LOC100132062 in human CRC tissues were notably higher compared with normal tissues (Fig. S1). The transfection of siRNAs targeting SLMO2-ATP5E and LOC100132062 decreased the expression of these IncRNAs, as confirmed by RT-qPCR assays (Fig. S2). However, the depletion of SLMO2-ATP5E and LOC100132062 had no obvious effects on CRC cell proliferation (Fig. S3), as shown by the colony-forming assays.

Subsequently, the effects of SCARNA9L on CRC progression were studied. Firstly, high mRNA expression 

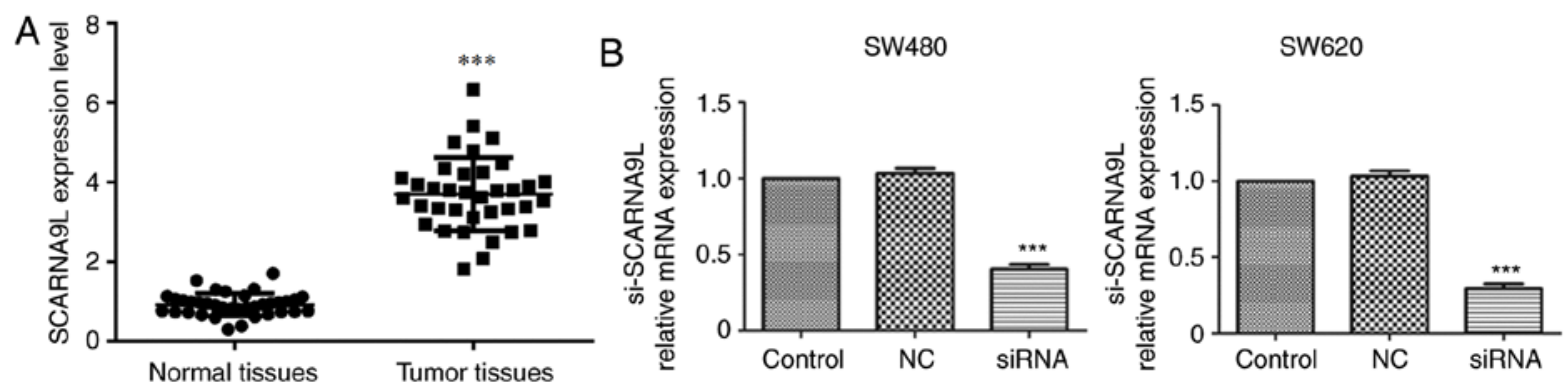

Figure 4. SCARNA9L expression is higher in human CRC tissues and effectively decreased in both SW480 and SW620 CRC CRC cells following the transfection of the corresponding siRNAs. (A) RT-qPCR assays showed notably high expression of SCARNA9L in 36 human tumor tissues compared with normal tissues. Data were analyzed using paired t-test. ${ }^{* * *} \mathrm{P}<0.001$. (B) RT-qPCR assays showed decreased expression levels of the lncRNA SCARNA9L, following the transfection with siRNA in SW480 and SW620 CRC CRC cells. Data were analyzed using one-way analysis of variance. ${ }^{* * *} \mathrm{P}<0.001 \mathrm{vs.} \mathrm{NC}$. CRC, colorectal cancer; NC, negative control; siRNA, small interfering RNA; RT-qPCR, reverse transcription-quantitative PCR.
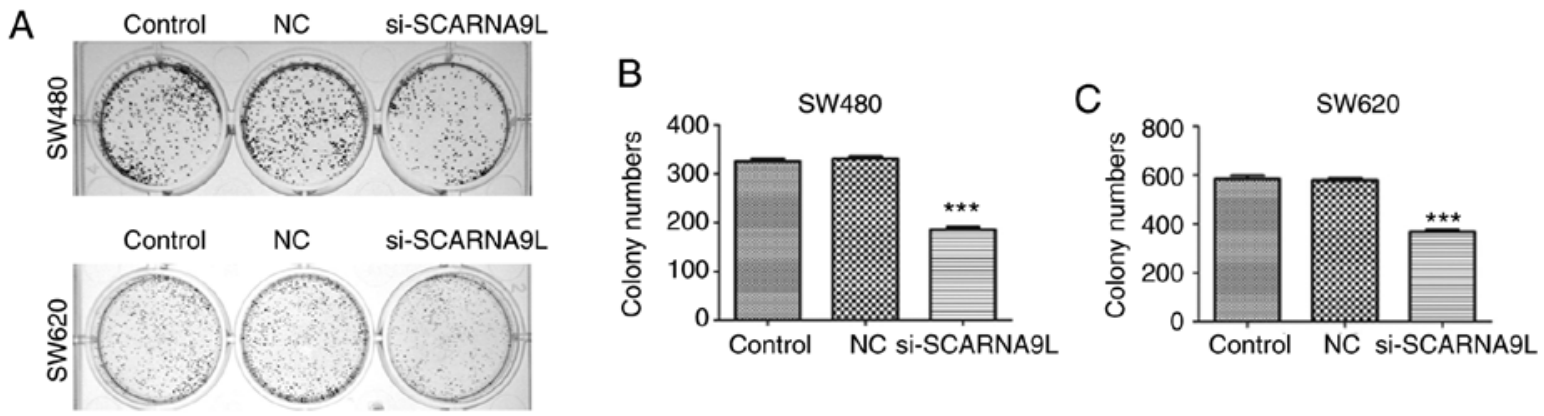

Figure 5. SCARNA9L facilitates the proliferation of colorectal cancer cells. (A) Colony-formation assays were performed in SW480 and SW620 cells, and representative images are shown. The number of colonies in the control group or the indicated siRNA-treated groups was manually counted in (B) SW480 and (C) SW620 cells. Data were analyzed using one-way analysis of variance. ${ }^{* * *} \mathrm{P}<0.001$ vs. NC. NC, negative control; siRNA, small interfering RNA.

levels of SCARNA9L were found in 36 human CRC tissues collected at The Second Hospital of Shandong University (Fig. 4A). Also, through RT-qPCR assays, it was found that the transfection of siRNAs targeting SCARNA9L effectively decreased the expression levels of the indicated lncRNAs in both SW480 and SW620 cells compared with the control group or the NC group (Fig. 4B). Interestingly, the depletion of SCARNA9L dramatically suppressed the proliferation of SW480 and SW620 cells, with a notable decrease in the number of colonies (Fig. 5A and B). The aforementioned results suggest that SCARNA9L could contribute to the proliferation of CRC CRC cells.

Depletion of SCARNA9L induces cell cycle arrest in CRC cells. The control of cell cycle is essential to maintain the proliferation of cells, and disruption of the cell cycle will result in abnormal proliferation and further promote tumorigenesis. Therefore, differences in the cell cycle distribution between cells with SCARNA9L knockdown and the control groups were detected. Notably, the data indicated that the ablation of SCARNA9L dramatically resulted in an increased percentage of cells at the $\mathrm{G}_{1}$ phase and a decreased proportion of cells at the $\mathrm{G}_{2} / \mathrm{M}$ phases (Fig. 6); suggesting the arrest of cell cycle at $\mathrm{G}_{1}$ phase. Thus, the ablation of SCARNA9L led to a significant cell cycle arrest and may further block CRC CRC cell proliferation. However, the depletion of SLMO2-ATP5E and LOC100132062 had no obvious effects on cell cycle (data not shown).
SCARNA9L contributes to CRC cell migration and invasion in vitro. Wound-healing and transwell assays were performed to evaluate the effects of SCARNA9L on the migration and invasion of SW480 and SW620 cells. Interestingly, the depletion of SCARNA9L significantly suppressed the extent of wound closure in both SW480 and SW620 cells (Fig. 7). Additionally, according to the transwell assays, depletion of SCARNA9L significantly inhibited the migration of SW480 and SW620 cells through membranes (Fig. 8). On the other hand, SLMO2-ATP5E and LOC100132062 had no effects on CRC cell migration (data not shown).

Overall, the lncRNA SCARNA9L contributes to cell migration and invasion of CRC in vitro.

\section{Discussion}

CRC is a common malignancy of the digestive tract, and its morbidity and mortality rates have notably increased (34). The occurrence of CRC without any obvious early symptoms also makes diagnosis difficult (35). Advanced CRC is highly metastatic and grows rapidly, leading to a poor prognosis (7). Effective biomarkers and therapeutic targets for CRC are urgently required (10). Notably, studies have indicated the widespread involvement of IncRNAs in cancer progression (19). Several IncRNAs are abnormally expressed in tumor tissues and correlate with the clinical features of patients (36). Additionally, these lncRNAs are usually involved in the regulation of cancer cell proliferation, migration or 

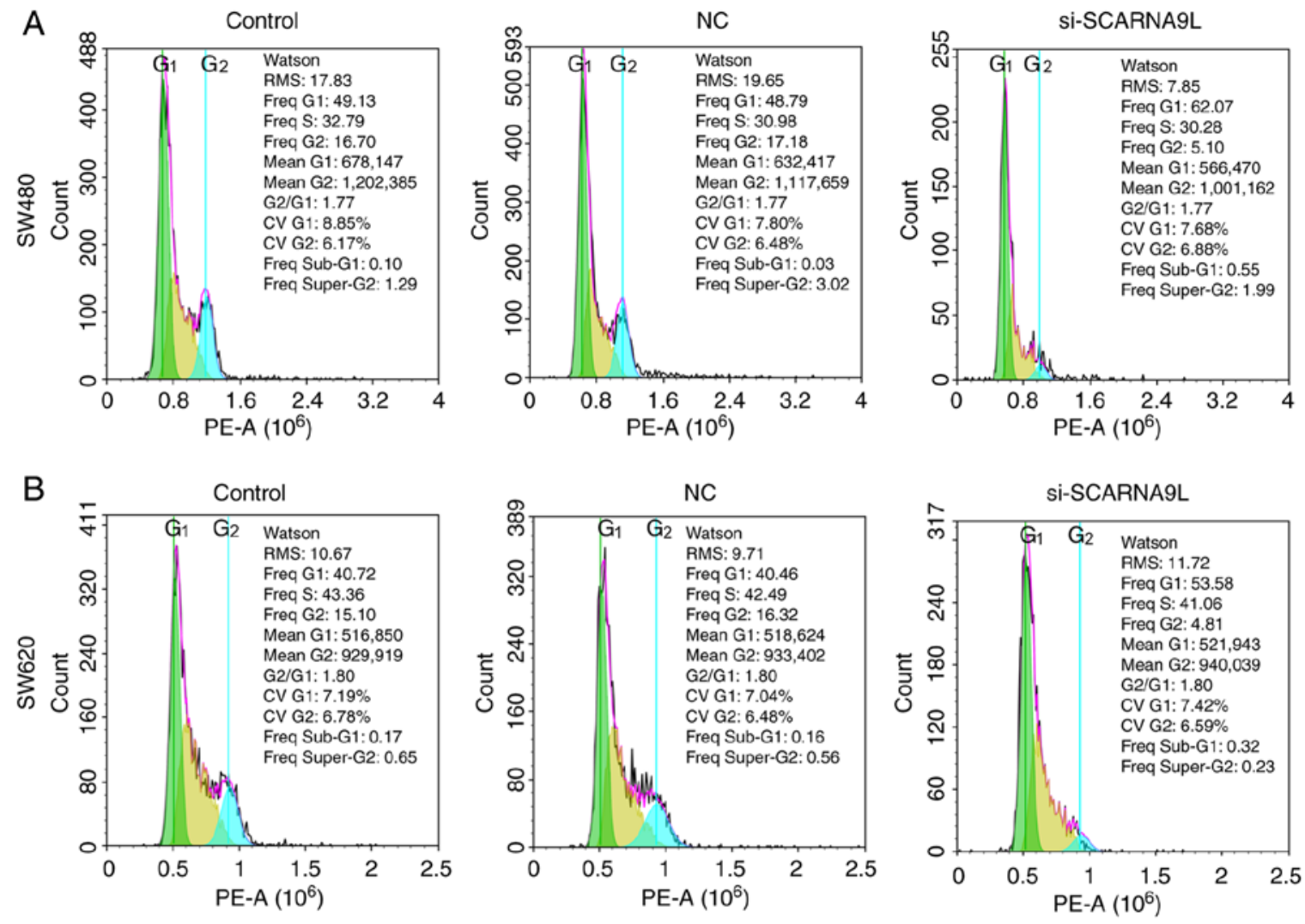

C
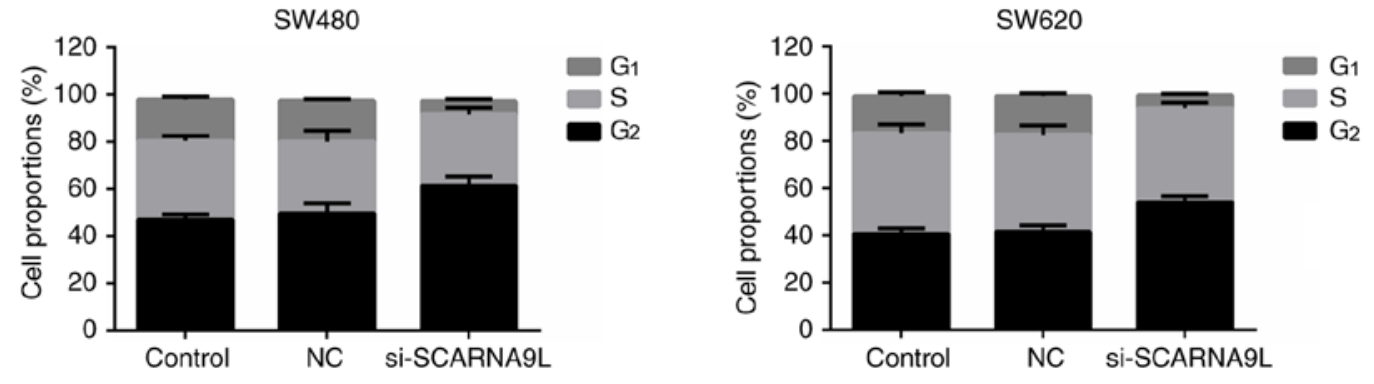

Figure 6. SCARNA9L is involved in the regulation of cell cycle in colorectal cancer cells. The analysis of the cell cycle distribution in control, NC or SCARNA9L siRNA-transfected (A) SW480 cells and (B) SW620 cells. (C) The proportion of SW480 or SW620 cells at each stage of the cell cycle. NC, negative control; siRNA, small interfering RNA; RMS, root mean square; Freq, frequency; CV, coefficient of variation.

apoptosis $(19,37,38)$. The present study identified three novel IncRNAs, SCARNA9L, SLMO2-ATP5E and LOC100132062, which were highly expressed in CRC tissues according to the microarray analysis. Furthermore, one of these lncRNAs, SCARNA9L, was found to affect CRC cell proliferation, migration and invasion. However, no significant effects were found in SLMO2-ATP5E, and LOC100132062 groups on cell cycle and migration (data not shown). These data indicate SCARNA9L as a novel and potential biomarker for CRC.

In the present study, SCARNA9L was demonstrated to be involved in CRC progression. Colony-formation assays showed impaired proliferation capacity, following the depletion of SCARNA9L. Flow cytometry assays confirmed that SCARNA9L affected the cell cycle of CRC cells in vitro. Furthermore, wound-closure and transwell assays found that SCARNA9L was also involved in the regulation of CRC cell migration. These are the first findings regarding the function of SCARNA9L in CRC progression. Combined with the present study, abnormal expression of SCARNA9L in CRC tissues was confirmed through microarray analysis and in vitro cell experiments, and various functions of CRC cells were affected. Thus, the present study suggests that SCARNA9L may serve as a potential biomarker, although further validation is required through mechanistic and animal studies, and clinical trials.

In addition to SCARNA9L, various IncRNAs have been identified to be abnormally expressed in CRC and affect its occurrence and development (19). The lncRNA LEF1-AS1 suppresses the progression of oral squamous cell carcinoma through the Hippo signaling pathway (39). Another study indicated that the IncRNA RP11 induces the dissemination of CRC cells via promoting the expression of ZEB1 (23). Similarly, the lncRNA MALAT1 also contributes to CRC progression via sponging miR-363-3p to mediate the expression of EZH2 (40). These studies, together with the findings of the present study, confirm the widespread involvement of IncRNAs in CRC development. However, the present study only found three lncRNAs that affected CRC proliferation, 
A

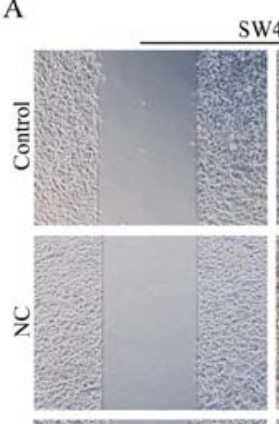

SW480
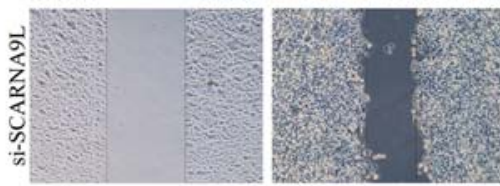

B
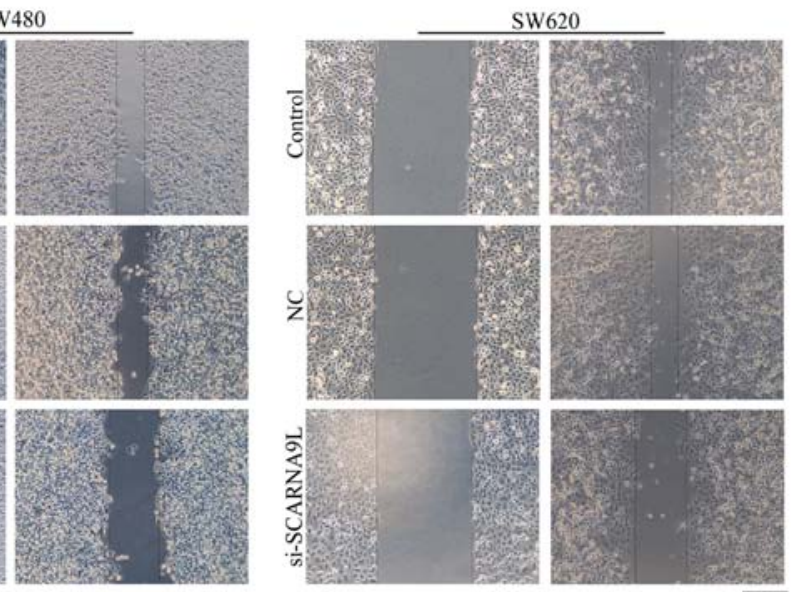

SW480

$\mathrm{C}$

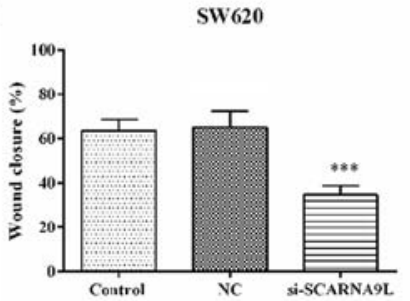

Figure 7. SCARNA9L contributes to colorectal cancer cell migration in vitro. (A) Knockdown of the lncRNA SCARNA9L resulted in reduced migration of SW480 and SW620 cells. Representative images show the migrated cells at the 0- and 24-h time points. The percentage of wound closure in (B) SW480 cells and (C) SW620 cells transfected with the indicated siRNAs. Scale bar, $1 \mathrm{~mm}$. Data were analyzed using one-way analysis of variance, ${ }^{* * *} \mathrm{P}<0.001 \mathrm{vs}$. NC. NC, negative control; siRNA, small interfering RNA.

A

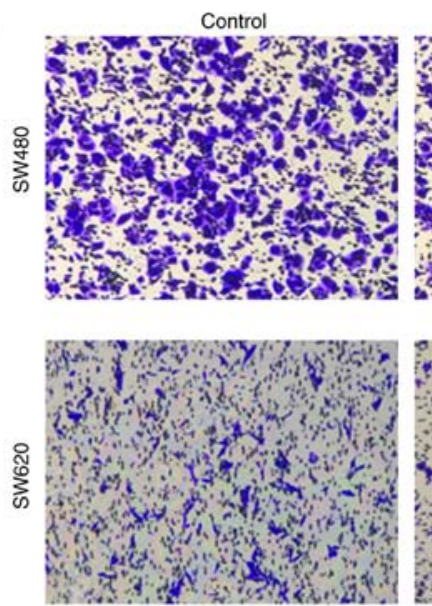

B

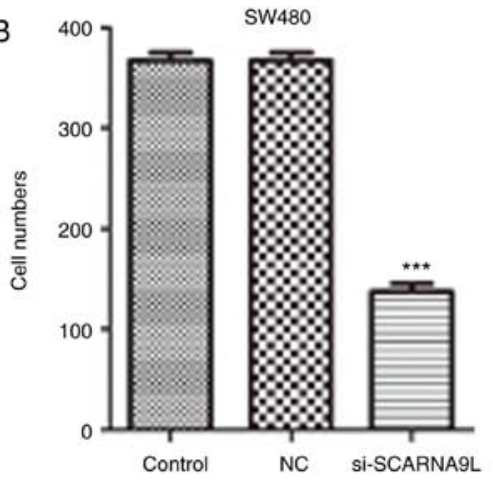

NC
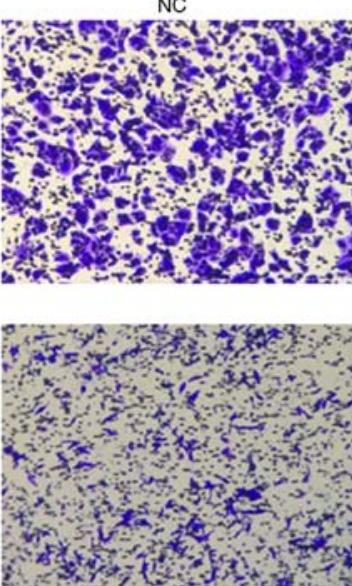

SI-SCARNA9L
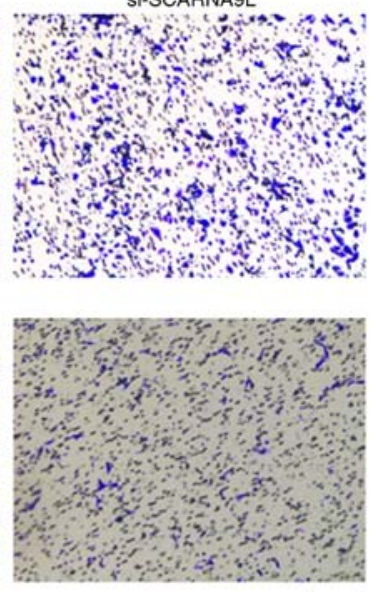

C

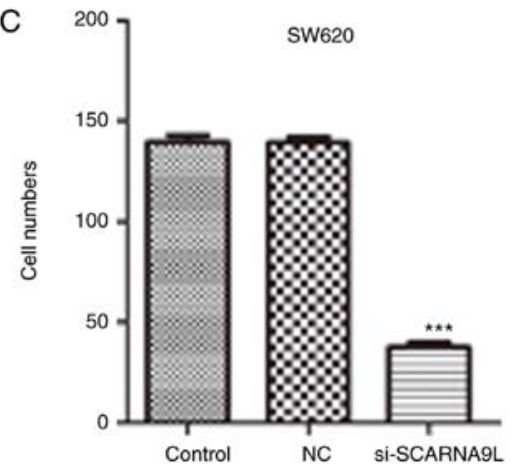

Figure 8. SCARNA9L promotes the migration of SW480 and SW620 cells in vitro. Transwell assays were performed using SW480 and SW620 cells transfected with NC or the indicated siRNAs. Representative images are shown in panel (A), and the migration was quantified by the number of stained (B) SW480 cells and (C) SW620 cells. Scale bar, $100 \mu \mathrm{m}$. The results were analyzed using one-way analysis of variance. ${ }^{* * * *} \mathrm{P}<0.001 \mathrm{vs.} \mathrm{NC.} \mathrm{NC,} \mathrm{negative} \mathrm{control;} \mathrm{siRNA,} \mathrm{small}$ interfering RNA. 
migration and invasion; the targets of these lncRNAs were not identified. Given that this study is the first to find that SCARNA9L can affect CRC progression, and there is no other evidence that these lncRNAs play key roles in the development of other tumors, the target genes of SCARNA9L and the mechanisms that affect tumor cell proliferation, migration and invasion should be further studied.

In the present study, SCARNA9L was identified to be involved in CRC progression through microarray analysis. Additionally, multiple signaling pathways and core lncRNAs that had the potential to affect CRC progression, and were obviously altered, were identified from the KEGG pathway analysis and coexpression network analysis. The ablation of SCARNA9L significantly restrained cell proliferation and induced cell cycle arrest in SW480 and SW620 cells. However, no obvious effects of SLMO2-ATP5E and LOC100132062 on CRC cell proliferation were identified in the present study. Furthermore, the present study identified SCARNA9L to significantly affect CRC cell migration. Thus, SCARNA9L is suggested as a novel and potential therapeutic target for the treatment of CRC.

\section{Acknowledgements}

Not applicable.

\section{Funding}

This study was supported by The China Postdoctoral Science Foundation (grant no. 2017M611177), The Shandong Province Natural Science Foundation (grant nos. ZR2017MH129 and ZR2013HL040), The China Natural Science Foundation (grant no. 81602578), and The Shandong Province Key Research and Development Plan (grant no. GG201710070085).

\section{Availability of data and materials}

All data generated or analyzed during this study are included in this published article. The datasets generated and/or analyzed during the current study are available in the Gene Expression Omnibus (GEO) repository (https://www.ncbi.nlm. nih.gov/geo/).

\section{Authors' contributions}

Conception and design, JC and WM. Development of methodology, JC, JZ, DH, WD, LH, LZ, BF, BL and WM. Acquisition of data (provided animals, acquired and managed patients, provided facilities), JC, JZ, DH, WD and BL. Analysis and interpretation of data (statistical analysis, biostatistics, computational analysis), HL and ZB. Writing, review, and/or revision of the manuscript, JC, BL and WM. Administrative, technical, or material support (reporting or organizing data, constructing databases), JC and JZ. All authors read and approved the final manuscript.

\section{Ethics approval and consent to participate}

This study was approved by the ethics committee at The Second Hospital of Shandong University (approval no. 201707003). Informed written consent was obtained from all patients.

\section{Patient consent for publication}

Not applicable.

\section{Competing interests}

The authors declare that they have no competing interests.

\section{References}

1. Stromberg U, Peterson S, Holmén A, Holmberg E, Hultcrantz R, Martling A and Nilbert M: Rational targeting of population groups and residential areas for colorectal cancer screening. Cancer Epidemiol 60: 23-30, 2019.

2. Jung C, Kim RS, Zhang H, Lee SJ, Sheng H, Loehrer PJ, Gardner TA, Jeng MH and Kao C: HOXB13 is downregulated in colorectal cancer to confer TCF4-mediated transactivation. Br J Cancer 92: 2233-2239, 2005.

3. Hosokawa A, Yamada Y, Shimada Y, Muro K, Hamaguchi T, Morita H, Araake M, Orita H and Shirao K: Prognostic significance of thymidylate synthase in patients with metastatic colorectal cancer who receive protracted venous infusions of 5-fluorouracil. Int J ClinOncol 9: 388-392, 2004.

4. Tian Y, Xu B, Yu G, Li Y and Liu H: Comorbidity and the risk of anastomotic leak in Chinese patients with colorectal cancer undergoing colorectal surgery. Int J Colorectal Dis 32: 947-953, 2017.

5. Guo X, Zhang C, Ma W, Tian F, Xu G, Han X, Sun P, Baklaushev VP, Bryukhovetskiy AS, Wang G, et al: Patterns of bone metastases in newly diagnosed colorectal cancer: A real-world analysis in the SEER database. Int J Colorectal Dis 34: 533-543, 2019.

6. Ahmed S, Johnson K, Ahmed $\mathrm{O}$ and Iqbal N: Advances in the management of colorectal cancer: From biology to treatment. Int J Colorectal Dis 29: 1031-1042, 2014.

7. Mele V, Sokol L, Kolzer VH, Pfaff D, Muraro MG, Keller I, Stefan Z, Centeno I, Terracciano LM, Dawson H, et al: The hyaluronan-mediated motility receptor RHAMM promotes growth, invasiveness and dissemination of colorectal cancer. Oncotarget 8: 70617-70629, 2017.

8. Yang KM, Park IJ, Lee JL, Kim CW, Yoon YS, Lim SB, Yu CS and Kim JC: Benefits of repeated resections for liver and lung metastases from colorectal cancer. Asian J Surg 43: 102-109, 2020.

9. Price TJ, Tang M, Gibbs P, Haller DG, Peeters M, Arnold D, Segelov E, Roy A, Tebbutt N, Pavlakis N, et al: Targeted therapy for metastatic colorectal cancer. Expert Rev Anticancer Ther 18: 991-1006, 2018.

10. Yamamoto $\mathrm{H}$ and Mori M: MicroRNAs as therapeutic targets and colorectal cancer therapeutics. Adv Exp Med Biol 937: 239-247, 2016.

11. Sun L, Zhang Y, Zhang Y, Gu Y, Xuan L, Liu S, Zhao X, Wang N, Huang L, Huang Y, et al: Expression profile of long non-coding RNAs in a mouse model of cardiac hypertrophy. Int J Cardiol 177: 73-75, 2014.

12. Hanly DJ, Esteller M and Berdasco M: Interplay between long non-coding RNAs and epigenetic machinery: Emerging targets in cancer? Philos Trans R Soc Lond B Biol Sci 373: 20170074 , 2018.

13. Dai L, Li J, Dong Z, Liu Y, Chen Y, Chen N, Cheng L, Fang C, Wang $\mathrm{H}$, Ji Y, et al: Temporal expression and functional analysis of long non-coding RNAs in colorectal cancer initiation. J Cell Mol Med 23: 4127-4138, 2019.

14. Zhao X, Yin H, Li N, Zhu Y, Shen W, Qian S, He G, Li J and Wang X: An integrated regulatory network based on comprehensive analysis of mRNA expression, gene methylation and expression of long non-coding RNAs (lncRNAs) in myelodysplastic syndromes. Front Oncol 9: 200, 2019.

15. Zhang Z: Long non-coding RNAs in Alzheimer's disease. Curr Top Med Chem 16: 511-519, 2016.

16. Hanson A, Wilhelmsen D and DiStefano JK: The role of long non-coding RNAs (lncRNAs) in the development and progression of fibrosis associated with nonalcoholic fatty liver disease (NAFLD). Noncoding RNA 4: E18, 2018.

17. Zhang P, Cao L, Zhou R, Yang $X$ and Wu M: The lncRNA Neat promotes activation of inflammasomes in macrophages. Nat Commun 10: 1495, 2019. 
18. Chandra Gupta S and Nandan Tripathi Y: Potential of long non-coding RNAs in cancer patients: From biomarkers to therapeutic targets. Int J Cancer 140: 1955-1967, 2017.

19. Xie X, Tang B, Xiao YF, Xie R, Li BS, Dong H, Zhou JY and Yang SM: Long non-coding RNAs in colorectal cancer. Oncotarget 7: 5226-5239, 2016.

20. Zhou Y, Gong B, Jiang ZL, Zhong S, Liu XC, Dong K, Wu HS, Yang HJ and Zhu SK: Microarray expression profile analysis of long non-coding RNAs in pancreatic ductal adenocarcinoma. Int J Oncol 48: 670-680, 2016.

21. Wen J, Wang H, Dong T, Gan P, Fang H, Wu S, Li J, Zhang Y, Du R and Zhu Q: STAT3-induced upregulation of lncRNA ABHD11-AS1 promotes tumour progression in papillary thyroid carcinoma by regulating miR-1301-3p/STAT3 axis and PI3K/AKT signalling pathway. Cell Prolif 52: e12569, 2019.

22. Zhang P, Dong Q, Zhu H, Li S, Shi L and Chen X: Long non-coding antisense RNA GAS6-AS1 supports gastric cancer progression via increasing GAS6 expression. Gene 696: 1-9, 2019.

23. Wu Y, Yang X, Chen Z, Tian L, Jiang G, Chen F, Li J, An P, Lu L, Luo $\mathrm{N}$, et al: $\mathrm{m}^{6} \mathrm{~A}$-induced lncRNA RP11 triggers the dissemination of colorectal cancer cells via upregulation of Zeb1. Mol Cancer 18: 87, 2019.

24. Barresi V, Trovato-Salinaro A, Spampinato G, Musso N, Castorina S, Rizzarelli E and Condorelli DF: Transcriptome analysis of copper homeostasis genes reveals coordinated upregulation of SLC31A1,SCO1, and COX11 in colorectal cancer. FEBS Open Bio 6: 794-806, 2016.

25. Barresi V, Cinnirella G, Valenti G, Spampinato G, Musso N, Castorina $S$ and Condorelli DF: Gene expression profiles in genome instability-based classes of colorectal cancer. BMC Cancer 18: 1265, 2018.

26. Condorelli DF, Spampinato G, Valenti G, Musso N, Castorina S and Barresi V: Positive caricature transcriptomic effects associated with broad genomic aberrations in colorectal cancer. Sci Rep 8: 14826, 2018.

27. Yang Y, Zhao Z, Xie CW and Zhao Y: Dual-targeting liposome modified by glutamic hexapeptide and folic acid for bone metastatic breast cancer. Chem Phys Lipids 228: 104882, 2020.

28. Loennstedt I and Speed P: Replicated microarray data. Statistica Sinica 12: 31-46, 2001.
29. Bauer S, Gagneur J and Robinson PN: GOing Bayesian: Model-based gene set analysis of genome-scale data. Nucleic Acids Res 38: 3523-3532, 2010.

30. Benjamini Y and Yekutieli D: The control of the false discovery rate in multiple testing under dependency. Annals of Statistics 29: 1165-1188: 2001

31. Best D and Roberts E: Algorithm AS 89: The upper tail probabilities of Spearman's rho. Applied Statistics 24: 377-379, 1975

32. Kanehisa M, Araki M, Goto S, Hattori M, Hirakawa M, Itoh M, Katayama T, Kawashima S, Okuda S, Tokimatsu T and Yamanishi Y: KEGG for linking genomes to life and the environment. Nucleic Acids Res 36: D480-D484, 2008.

33. Livak KJ and Schmittgen TD: Analysis of relative gene expression data using real-time quantitative PCR and the 2(-Delta Delta $\mathrm{C}(\mathrm{T}))$ method. Methods 25: 402-408, 2001

34. Mateo-Lozano S, Bazzocco S, Rodrigues P, Mazzolini R, Andretta E, Dopeso H, Fernández Y, Del Llano E, Bilic J, Suárez-López L, et al: Loss of the EPH receptor B6 contributes to colorectal cancer metastasis. Sci Rep 7: 43702, 2017.

35. Cerdán-Santacruz C, Cano-Valderrama O, Cárdenas-Crespo S, Torres-García AJ and Cerdán-Miguel J: Colorectal cancer and its delayed diagnosis: Have we improved in the past 25 years? Rev Esp Enferm Dig 103: 458-463, 2011.

36. Bai J, Yao B, Wang L, Sun L, Chen T, Liu R, Yin G, Xu Q and Yang W: IncRNA A1BG-AS1 suppresses proliferation and invasion of hepatocellular carcinoma cells by targeting miR-216a-5p. J Cell Biochem 120: 10310-10322, 2019.

37. Rossi MN and Antonangeli F: LncRNAs: New players in apoptosis control. Int J Cell Biol 2014: 473857, 2014.

38. Gioia R, Drouin S, Ouimet M, Caron M, St-Onge P, Richer C and Sinnett D: Lncrnasdownregulated in childhood acute lymphoblastic leukemia modulate apoptosis, cell migration, and DNA damage response. Oncotarget 8: 80645-80650, 2017

39. Jin Q, Dai Y, Wang Y, Zhang S and Liu G: High kinesin family member 11 expression predicts poor prognosis in patients with clear cell renal cell carcinoma. J ClinPathol 72: 354-362, 2019.

40. Xie JJ, Li WH, Li X, Ye W and Shao CF: LncRNA MALAT1 promotes colorectal cancer development by sponging miR-363-3p to regulate EZH2 expression. J Biol Regul Homeost Agents 33: 331-343, 2019. 